\title{
REYNOLDS AND FROUDE NUMBER SCALING IN STABLY-STRATIFIED FLOWS
}

\author{
Stephen M. de Bruyn Kops \\ University of Massachusetts \\ Amherst, MA 01003
}

James J. Riley

University of Washington

Seattle, WA 98195

Kraig B. Winters

Scripps Institution of Oceanography, UCSD

La Jolla, CA 92037

\begin{abstract}
High resolution direct numerical simulations and analytical scaling arguments are used to understand the dynamics of turbulence strongly affected by stable stratification, and to estimate the Reynolds number above which laboratory and numerical experiments of these flows must be conducted in order for the results to scale up to geophysical cases. For low Froude number $F_{h}$ and high enough Reynolds number $R_{h}$, where $F_{h}$ and $R_{h}$ are based upon the horizontal integral scale of the motion, it is found that the simulated flows become quasi-horizontal with high vertical shearing of the horizontal velocity; this in turn leads to locally low Richardson numbers and susceptibility to shear instabilities. This occurs even though the nominal Richardson number is greater than order one. Kelvin-Helmholtz instabilities were observed to be one pathway to turbulence in the simulated flows. It is estimated that instabilities and turbulence in these flows will occur if $R e_{h} F_{h}^{2}>\mathcal{O}(1)$, suggesting that this criterion need be met for the laboratory and numerical experiments to be useful in modeling geophysical scale flows.
\end{abstract}

Keywords: turbulence, stable stratification, Reynolds number, Froude number 


\section{Introduction}

Stably-stratified flows occur in most of the ocean below the mixed layer, in the atmosphere above the tropopause, and in the nocturnal atmospheric boundary layer over land. Turbulence in these flows is of great interest because it affects, e.g., climate, weather, and contaminant dispersion; it often decays due to the lack of a continuous source of energy. Examples of this include turbulence due to the breakdown of a propagating internal wave, turbulence resulting from a local shear instability, and turbulence due to wakes of islands, mountains, or vehicles.

Laboratory experiments by, e.g., Spedding et al., 1996a, Spedding et al., 1996b, Chomaz et al., 1993, and Bonnier et al., 2000, show that, when the effects of the stratification become strong, i.e., when the Froude number is of order one or less, the dynamics of stratified flows change dramatically and quasi-horizontal vortices form. As the turbulence develops without energy input from the mean flow, both the Reynolds and Froude numbers decrease. There results a competition in which Reynolds number effects tend to cause turbulence and gravity tends to suppress it so that turbulence erupts in patches and then decays. In this regime, the dynamics of the turbulence are not well understood and predictive methods work poorly. This is an area of geophysical turbulence where predictive methods are the weakest.

In the laboratory experiments just cited, the Reynolds numbers become low by the time the stratification effects become strong. In geophysical flows, however, the Reynolds number is very high, which leads to several open questions: (1) do the laboratory experiments scale up to the geophysical case, (2) do quasi-horizontal vortices exists in the geophysical case, and (3) if they do exist, what are their dynamics. In this paper, we address questions 1 and 3 .

\section{Numerical Simulations}

To address the open questions just raised, we use high resolution direct numerical simulations (DNSs) in conjunction with scaling analysis. The two flows studied via DNS are Taylor-Green vortices and a "vortex street." The Taylor-Green vortex simulations (Riley and de Bruyn Kops, 2003) are analogous the grid experiments of Fincham et al., 1996. They were initialized with perturbed Taylor-Green vortices (Taylor and Green, 1937) and run with nominal Reynolds and Froude numbers in the range 200 to 6400 and 1.4 to 4 , respectively. This resulted in simulated flows ranging from barely turbulent to highly turbulent, and local stratification effects ranging from extremely strong to moderate. The "vortex street" simulations are analogous to late wake experiments (Spedding et al., 1996a). As with the Taylor-Green simulations, they were run for 
a range of Reynolds and Froude numbers. All the simulations were computed with $512^{3}$ or $512 \times 512 \times 256$ grid points using massively parallel pseudo-spectral codes.

Quantitative results from the Taylor-Green simulations are reported by Riley and de Bruyn Kops, 2003. In summary, the simulated flows behave as expected in that the horizontal length scales grow and the vertical scales decrease with time, resulting in a quasi-horizontal flow with strong vertical shearing of the horizontal velocity. To better understand how this shearing leads to unstable patches and turbulence, we analyze the flow qualitatively here beginning with Fig. 1, in which the vertical velocity is shown on a horizontal plane through the region of maximum shear. The white line in each panel of the figure connects two material points as they move in time. We see that the flow is fairly calm at scaled time $t=17.5$, but that an instability occurs in the the vicinity of the white line by $t=20$. By $t=22.5$, the area around the line is engulfed in turbulence.

By slicing the domain vertically through the white line, the dynamics of this particular instability can be studied. In Fig. 2, the total density is shown on a vertical plane through the white line at $t=21.5$. The classic Kelvin-Helmholtz roll-ups visible in the image give evidence of that mechanism being a pathway to the turbulent bursts that occur in these simulations.

A similar analysis is made of the vortex street simulations. As in the Taylor-Green case, the simulated flow evolves as expected based on laboratory results and theoretical arguments. By slicing the flow with a vertical plane through an unstable region, the instability mechanism is again observed to be Kelvin-Helmholtz. This can be seen in Fig. 3.

\section{Scaling Analysis}

From these simulation results, in conjunction with theoretical and experimental results by other researchers, there emerges an explanation for instabilities and turbulence in strongly stratified flows. When the Froude number based on the horizontal integral scale of motion, $F_{h}$, is of order one or less, growth of the horizontal scales and nonlinear interactions cause continual development of small vertical scales. This occurs even though the initial buoyancy Richardson number is much larger than unity. The resulting regions of high shear and low local Richardson number make the flow susceptible to Kelvin-Helmholtz instabilities. For these instabilities to occur, however, the Reynolds number must be sufficiently high.

To estimate the required Reynolds number, we begin with the condition that the local buoyancy Richardson number $R i=N^{2} /\left(u_{h}^{\prime 2} / L_{z}^{2}\right)$ is of 
$\mathrm{t}=17.5$

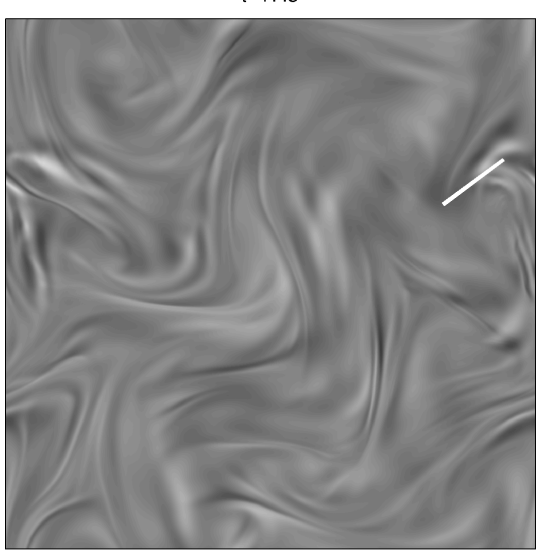

$t=21.5$

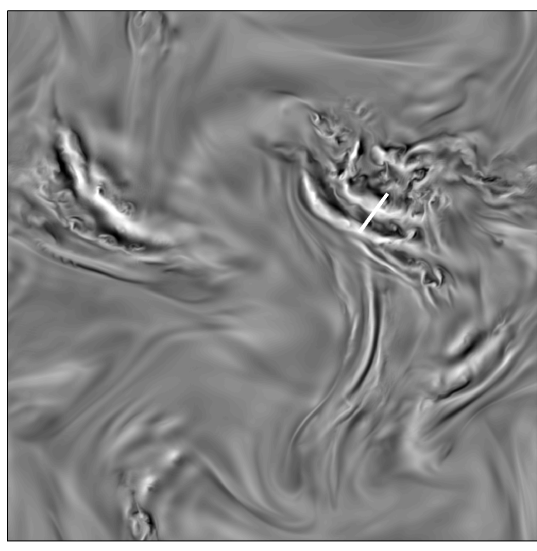

$\mathrm{t}=20$
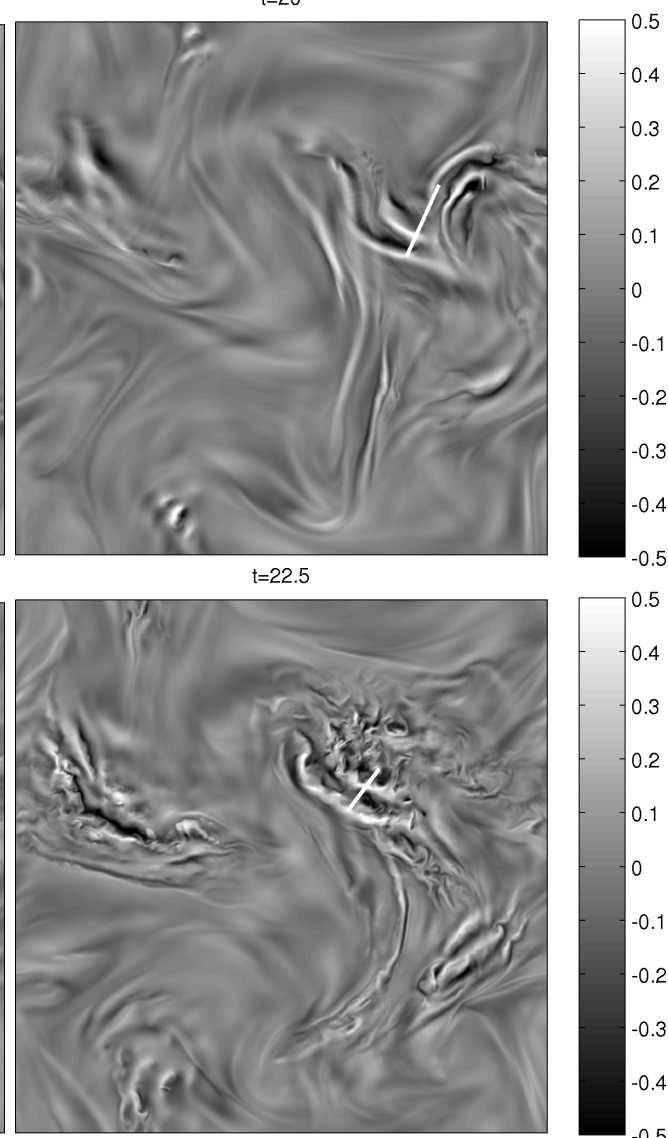

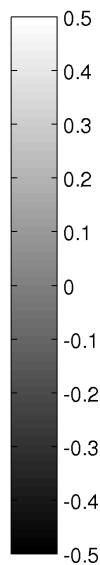

Figure 1. A horizontal slice through the vertical velocity field at the plane of maximum shear at four different times in a Taylor-Green simulation with Froude number 2 and Reynolds number 3200. The white bar connects two material points that move with time. The points start in a region of relative calm, experience an instability, and end up in a turbulent patch.

Figure 2. The total density on a vertical cut aligned with the white bar in Fig. 1, $t=21.5$. 

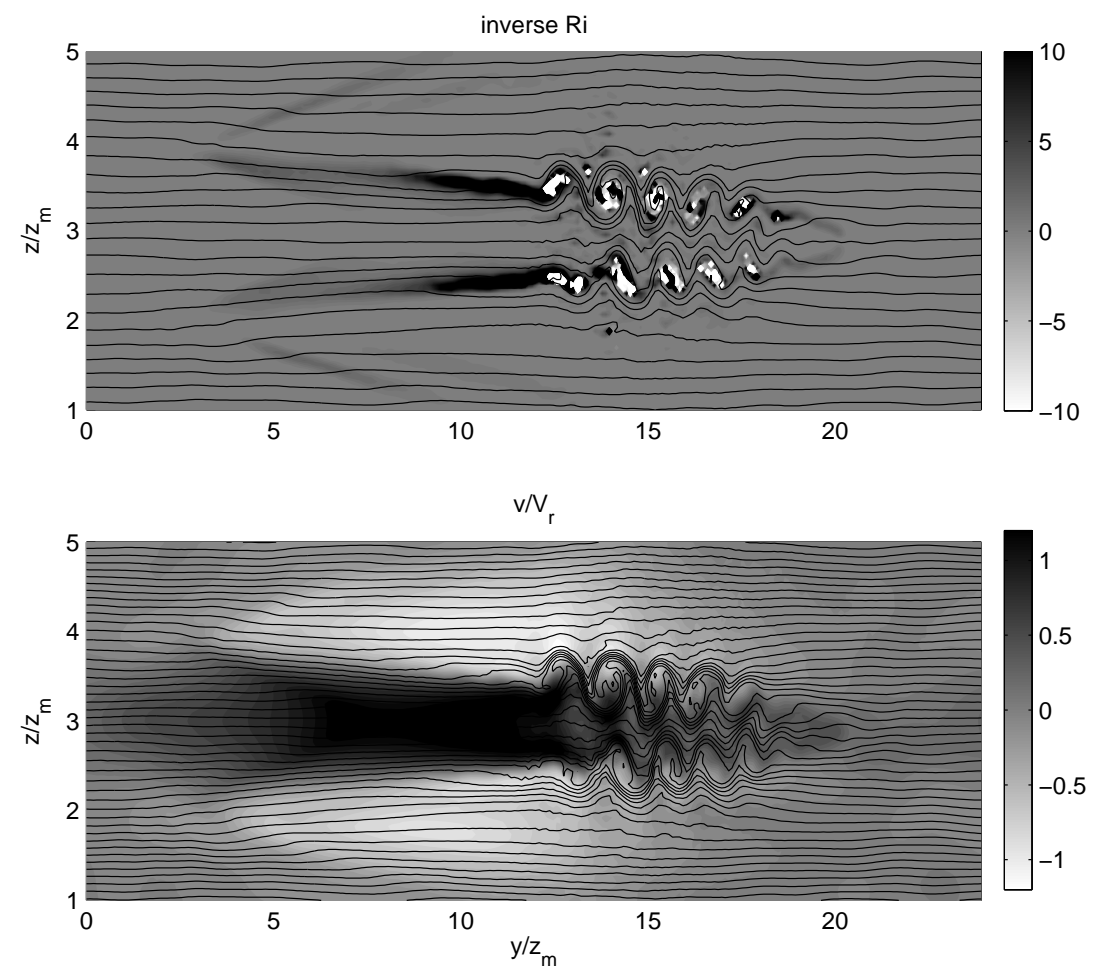

Figure 3. Upper panel: Inverse Richardson number in span-wise vertical plane from vortex street simulation. Values greater than 4 indicate potential for shear instability. Values less than zero indicate overturned density surfaces. Lower panel: spanwise velocity normalized by initial horizontal swirling velocity of vortices. Dark regions indicate flow to the right, light regions indicate flow to the left.

order one or less. Here, $N$ is the buoyancy frequency, $L_{z}$ is a length scale of the vertical shearing (Taylor scale), and $u_{h}^{\prime 2}$ is the mean square horizontal velocity. By assuming that the advection and viscous dissipation terms in the kinetic energy equation are of the same order of magnitude, we can estimate that the denominator is of order $u_{h}^{\prime 3} / \nu L_{h}$, where $L_{h}$ is the characteristic length of the horizontal motions. This leads to the Reynolds number criteria

$$
R e_{h}=\frac{u_{h}^{\prime} L_{h}}{\nu}>\mathcal{O}\left(\frac{1}{F_{h}^{2}}\right)
$$

where $F_{h}=u^{\prime} / N L_{h}$. A more detailed justification for this scaling is given by Riley and de Bruyn Kops, 2003.

It is important to note that while Kelvin-Helmholtz instabilities are observed in the simulated flows, there may be other pathways to turbu- 
lence. The classic roll-ups shown in this paper are quite clear, but in simulations in the same series but with different nominal Reynolds and Froude numbers, turbulent patches appear with no obvious instability mechanism. Billant and Chomaz, 2000, have shown that, in model problems with strong stable stratification, the quasi-horizontal flows can also be subject to a "zig-zag" instability

\section{Conclusions}

High resolution direct numerical simulations and analytical scaling arguments have been used to understand the dynamics of stably-stratified flows and to estimate the Reynolds number above which laboratory and numerical experiments of these flows must be conducted in order for the results to scale up to geophysical cases. Consistent with the results from other researchers, the simulated flows become quasi-horizontal with high vertical shearing of the horizontal velocity and low local Richardson numbers. Kelvin-Helmholtz instabilities were observed to be a pathway to turbulence in the simulated flows. Scaling arguments suggest that instabilities and turbulence can be expected in these flows provided that $R e_{h} F_{h}^{2}>\mathcal{O}(1)$.

This research was funded by the U.S. Office of Naval Research, Grant No. N00014-00-1-0752, managed by Dr. L. Patrick Purtell; grants of high performance computer (HPC) time were provided by the Arctic Region Supercomputing Center and the Army HPC Research Center.

\section{References}

Billant, P. and Chomaz, J. M. (2000). Three-dimensional stability of a vertical columnar vortex pair in a stratified fluid. J. Fluid Mech., 419:65-91.

Bonnier, M., Eiff, O., and Bonneton, P. (2000). On the density structure of far-wake vortices in a stratified fluid. Dyn. Atmos. Oceans, 31:117-137.

Chomaz, J.-M., Bonneton, P., Butet, A., and Hopfinger, E. J. (1993). Vertical diffusion of the far wake of a sphere moving in a stratified fluid. Phys. Fluids A, 5:2799.

Fincham, A. M., Maxworthy, T., and Spedding, G. R. (1996). Energy dissipation and vortex structure in freely decaying, stratified grid turbulence. Dyn. Atmos. Oceans, 23:155-169.

Riley, J. J. and de Bruyn Kops, S. M. (2003). Dynamics of turbulence strongly influenced by buoynacy. Phys. Fluids. (submitted).

Spedding, G. R., Browand, F. K., and Fincham, A. M. (1996a). The long-time evolution of the initially-turbulent wake of a sphere in a stable stratification. Dyn. Atmos. Ocean., 23:171.

Spedding, G. R., Browand, F. K., and Fincham, A. M. (1996b). Turbulence, similarity scaling and vortex geometry in the wake of a towed sphere in a stably stratified fluid. J. Fluid Mech., 314:53.

Taylor, G. I. and Green, A. E. (1937). Mechanism of the production of small eddies from large ones. Proc. Roy. Soc. A, 158:499-521. 